Liliana Ruiz Gutiérrez*

\title{
La Santamaría, una plaza para todos ${ }^{1}$
}

\author{
La Santamaría, the arena for everyone
}

Cómo citar:

Ruiz Gutiérrez, L. (2017). La Santamaría, una plaza para todos. Designia, 4(2), 83-105.

${ }^{1}$ Artículo resultado de investigación científica y tecnológica, elaborado a partir de los productos obtenidos en la consultoría para la elaboración del proyecto urbano y arquitectónico para la Plaza de Toros La Santamaría, a cargo del Instituto Distrital de Patrimonio Cultural de Bogotá.

*Arquitecta restauradora con experiencia en patrimonio cultural, gestión urbana, trabajo comunitario en sectores de

interés cultural y zonas rurales con población residente y en condición de vulnerabilidad, así como en la formulación y articulación de políticas culturales vinculadas con el ordenamiento territorial. Docente universitaria en temas de intervención en patrimonio cultural, historia de la ciudad y urbanismo.

Email.sliliruiz@gmail.com-arqlilianaruizg@gmail.com

\section{Palabras clave:}

Ciudad histórica, conservación de monumentos, bien cultural, técnica de conservación.

\section{Key words:}

This paper is based on research which analysed the Santamaria's Bullring from different perspectives (historical, technical construction, use, social representativeness and future

Recibido: 8/06/2016

Aceptado: 29/11/2016

\section{Resumen:}

Este artículo presenta una investigación sobre la Plaza de Toros de Santamaría, en la cual el inmueble se abarcó desde distintos puntos de vista (histórico, técnico-constructivo, uso, representatividad social y sostenibilidad futura). Se emplearon metodologías investigativas para bienes de interés cultural y se recurrió a estudios previos, análisis de laboratorio y otros especializados en arquitectura, restauración, sistemas constructivos y estructurales para determinar técnicamente su estado de conservación. Promovida por el ganadero Ignacio Sanz de Santamaría, su construcción se inició en 1928, primero con un estilo simple propio de los primeros desarrollos de la arquitectura en concreto que posteriormente fue complementado con un estilo neomudéjar en sus fachadas, siendo en ese entonces el equipamiento cultural más grande construido en Bogotá y hoy, 
reconocido como una de las edificaciones más emblemáticas de la ciudad, ejemplificando la arquitectura de principios de siglo XX. Declarada monumento nacional (hoy bien de interés cultural de la Nación) en 1984 y localizada en las inmediaciones del Centro Internacional y de otros edificios patrimoniales, se constituye en un ícono para Bogotá. A partir de 2012 las corridas de toros fueron restringidas allí en medio de un debate sobre esta práctica y en 2013 se cerró al público debido a sus problemas estructurales y riesgos para la seguridad humana. El proyecto de intervención busca recuperar sus elementos arquitectónicos representativos, dotarla de condiciones de última tecnología para diferentes actividades y generar un espacio público en su área inmediata que la integre con otros bienes culturales y potencie dinámicas urbanas.

\section{Abstract:}

This paper is based on research which analysed the Santamaria's Bullring from different perspectives (historical, technical construction, use, social representativeness and future sustainability). Research methods for cultural buildings were used, as well as prior surveys, laboratory studies and specialized analysis in architecture, restoration, construction and structural systems, pointing to technically determine the conservation conditions. Promoted by the rancher Ignacio Sanz de Santamaria, this bullring began to be constructed in 1928, first with a simple style of the first concrete buildings that was later modificated with ag a neo-mudejar style and it became the largest cultural venue in Bogota. It is nowadays one of the city's most significant constructions exemplifying the early 20th century architecture. It was declared a cultural heritage building in 1984 and located in the International Center and other heritage buildings, is an icon for Bogota.However, it was closed in 2013 due to structural problems and risks for human safety. In addition, bullfight was banned to be performed there in 2012 amid a controversy on this practice. The intervention project aims to recover the arena's most representative architectonical elements along to equip it with state-of-the-art technology in order to enable it for different activities. Moreover, a public space it is expected to be generated in the surroundings as a way of integration with other cultural buildings, thereby strengthening multiple urban dynamics. 


\section{INTRODUCCIÓN}

La protección del patrimonio cultural es una estrategia que se dio en Bogotá a lo largo del siglo XX y poco a poco ha empezado a dar sus frutos, tras más de 40 años de investigaciones y proyectos de restauración que se han encargado de cambiar la cara de bienes de interés cultural y abrirlos al público para su disfrute colectivo.

El presente documento describe el proyecto de restauración integral para la Plaza de Toros La Santamaría y su entorno inmediato, elaborado entre 2012 y 2013 por el Instituto Distrital de Patrimonio Cultural de la Alcaldía Mayor de Bogotá, en el marco del Plan de Revitalización del Centro Tradicional. Se buscó intervenir esta pieza urbana ubicada en el borde norte del centro histórico, para incorporarla a las dinámicas actuales del sector.

El problema de investigación se desarrolló a partir de la necesidad de identificar las condiciones de la Plaza y dotarla con los elementos técnicos requeridos para fortalecerla como un equipamiento cultural, pues así ha sido definida por el Plan de Ordenamiento Territorial (POT) de la ciudad. Se tuvo presente que se trata de un bien de interés cultural de la nación, cercano a otros edificios de valor patrimonial.

La metodología utilizada fue planteada en los términos establecidos para la consultoría contratada, en los cuales se indicaron las técnicas de elaboración para un proyecto de restauración que requiere seguir los lineamientos definidos por la Ley General de Cultura y los planteados en la reglamentación internacional para la intervención de un bien de interés cultural nacional. Fue preciso considerar distintos componentes (arquitectónico, estructural, arqueológico e histórico) al 
igual que el carácter de la Plaza como pieza estratégica para el centro bogotano. Se realizó entonces un análisis de sus condiciones en el sector y de la edificación en sí misma, su proceso constructivo, vulnerabilidad sísmica, afectación por usos diversos y sus patologías, para definir las pautas a seguir en aras de recuperarla como hito generador de identidad de Bogotá y parte integral de su memoria.

La investigación se centró en dos aspectos. Por una parte, para el diagnóstico y la propuesta de intervención de la edificación y su entorno, se revisó material de archivo histórico y fotográfico, junto a otros estudios previamente elaborados. También se efectuaron varios pasos: exámenes de laboratorio, levantamiento arquitectónico, calificación del estado actual de conservación, estudio de vulnerabilidad sísmica e identificación de las condiciones de las redes hidrosanitarias, eléctricas y especiales. Se evaluaron los usos pasados y el potencial de desarrollo futuro. Para el entorno inmediato se analizaron determinantes de diseño y se consultó cartografía urbana y aerofotografía histórica.

El segundo aspecto, asociado al proyecto de intervención, abordó la Plaza desde distintos componentes (urbano, arquitectónico, sostenibilidad, estructural y de gestión) con el fin de dotar a este bien de las condiciones adecuadas de acuerdo con las necesidades presentes de la ciudad y del contexto donde se ubica.

\section{CIUDAD Y TAUROMAQUIA}

Al hablar de toros, de inmediato la mente nos lleva a una tradición heredada de España que ha acompañado a algunos de sus países vecinos y a buena parte de los latinoamericanos. Es importante conocer cómo arribó esta práctica a la península Ibérica y luego al "Nuevo Mundo" hasta llegar a ser recurrente e influyente en la conformación física de sectores urbanos. "La fiesta brava", como es conocida, está vinculada al periodo Al-Andalus, cuando los musulmanes ocuparon la Península Ibérica durante la Edad Media. Sus orígenes se sitúan en las explanadas abiertas próximas a las puertas de las ciudades, denominadas musaras, donde inicialmente se practicaban ejercicios de caballería y de carácter militar. En algunas oportunidades, allí tenían lugar mercados y otras actividades de intercambio comercial. 
En ciertas ocasiones, las musaras coincidían con las musallas, también explanadas, con un muro orientado a La Meca, en las cuales se reunía toda la comunidad musulmana de la ciudad con ocasión de determinados ritos religiosos. La musalla era, pues, una especie de mezquita abierta, un lugar, por tanto, ocasionalmente sagrado. Estos espacios son los que, después de la Reconquista, cambian su nombre por el de coso y los que a partir del siglo XV se constituyen en el escenario habitual de las corridas de toros (Pérez de Lama Halcón, 1994, p. 186).

Con el tiempo, las corridas empezaron a celebrarse al interior de las ciudades, para lo cual plazas y calles se adaptaban con barreras, graderías y corrales, todo de uso efímero, principalmente en época de fiestas religiosas y carnavales. En ciertos casos se habilitaban calles aledañas a los mataderos, que además de proveer fuentes de alimentación, se convertían así en espacios festivos antes del sacrificio.

\footnotetext{
Se obligaban a cerrar algunas calles y pasadizos con maderos. A aquel acontecimiento se van añadiendo otros que progresivamente van limitando la presencia del toro en la ciudad, para acabar disponiéndose, en 1533, que los toros se corriesen en la plaza, en la que se construiría con maderos una barrera que cerraba la salida (Pérez de Lama Halcón, 1994, p. 188).
}

En el Renacimiento los toros se convierten en un espectáculo urbano y la plaza mayor lo recibe como uno más de los eventos allí realizados y vinculados a la población. Durante el Barroco, en muchas ciudades españolas aparecen plazas ochavadas. Estos son espacios públicos octogonales con fachadas en todos sus costados que generan un conjunto unitario y armónico, dando una nueva imagen urbana que con frecuencia se adecuaban para celebraciones taurinas. 
El toreo abierto en la ciudad fue gradualmente haciéndose objeto de fuertes cuestionamientos por sus riesgos y accidentes para los habitantes. Ante esto, se vio la necesidad de diseñar un espacio cerrado destinado a tal actividad que evitara así el uso de plazas y plazoletas. Entre 1750 y 1850 en España se definieron normas y características para estas edificaciones. En algunas de ellas el uso taurino convivía con otros, como es el caso de la Plaza de Almadén, de 1757, un edificio de vivienda con un patio interior poligonal para espectáculos públicos, entre ellos los toros.

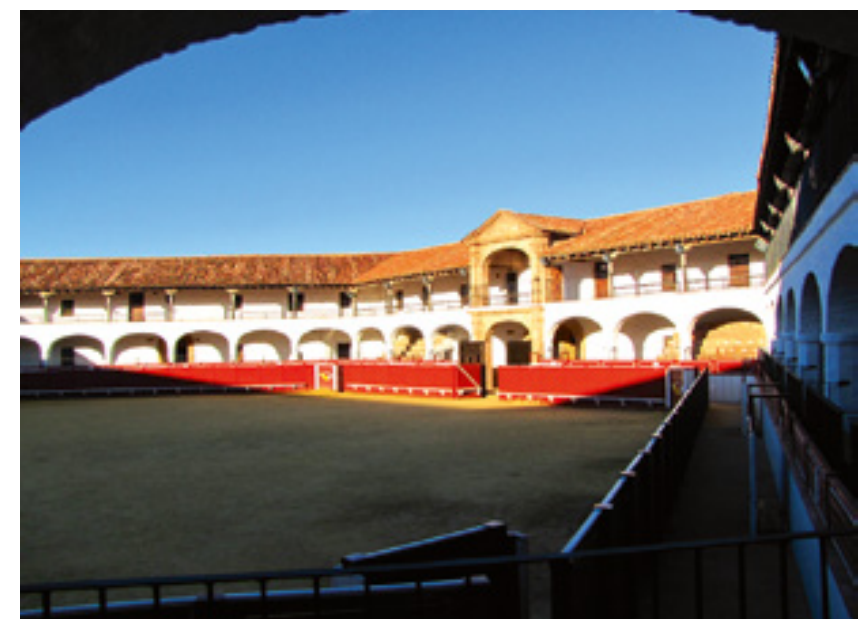

Figura 1. Interior de la Plaza de Almadén. Fuente: Liliana Ruiz Gutiérrez.

Durante este periodo también se construyen escenarios temporales de madera. Ya en el siglo XIX, las plazas de toros pasan a ser edificios públicos monumentales, especialmente de planta circular, grandes dimensiones, con una o varias portadas y una relación directa con el entorno donde se localizan. 
A lo largo del siglo XIX se construyen varias plazas y para principios del XX se implementa este modelo en las ciudades de América Latina, donde se levantan particularmente en las afueras, en una búsqueda por estimular nuevos desarrollos en áreas de ensanche. En su mayoría ocupan manzanas completas y se convierten en hitos por su volumetría y características formales. Además, su forma genera una conformación morfológica al definir parámetros urbanos singulares en sus entornos.

\begin{abstract}
A finales del siglo XIX este tipo de edificio, entre el espectáculo y la fiesta, está bien consolidado y es bien conocida su forma. El ancho de las crujías superiores reducido a un paso externo a los balcones; la seriación de un sector como sistema de componer el círculo; la imagen externa de arcos superpuestos; la sistemática disposición de sus ejes; la formalización simbólica de su puerta principal como "portada" para la presentación del nuevo edificio en la trama urbana; la relación dimensional y compositiva entre sus partes (ruedo, callejón, gradas, balcones, toriles y demás dependencias y servicios) van a ser a partir de ahora las constantes de un edificio que ya ha encontrado su forma y su imagen (Pérez de Lama Halcón, 1994, p. 215).
\end{abstract}

\title{
EL ESTILO NEOMUDÉJAR EN EL LENGUAJE TAURINO
}

El neomudéjar es un estilo decorativo usado esencialmente en fachadas para dignificar las áreas públicas de edificios, en el cual se destaca el ladrillo a la vista y los arcos tipo herradura. Surgió en España a finales del siglo XIX, en un intento por encontrar un estilo nacional a partir de la valoración del pasado histórico del país.

Se puede definir como un intento, de los tantos que hubo en esos años, para hallar en un momento de imaginada decadencia arquitectónica un estilo nacional. Una búsqueda que coincide con los efectos y resultados de los procesos de cambio auspiciados por el exilio de Isabel II y el inicio del Sexenio revolucionario (1868-1874) en el que se suceden un gobierno provisional, una monarquía democrática, una república federal unitaria y finalmente una restauración de la dinastía derribada; pero también por los efectos del auge de nacionalismos como el alemán y el italiano y el inicio del descubrimiento y valoración del pasado arqueológico e histórico del país (...) merece destacar la reivindicación que Amador de los Ríos hará, tras sus estudios, del mudéjar como estilo y arte propiamente hispánico (Checa Artazu, 2009). 
Entre los promotores del estilo, en especial en Madrid, se encuentran los arquitectos Agustín Ortiz de Villajos (1829-1902) y Emilio Rodríguez Ayuzo (1845-1891).

\footnotetext{
Estos técnicos vieron en el mudéjar el resultado artístico de la mezcla de cristianos y musulmanes, quienes concitaron una idea de unidad del solar hispánico en época medieval que se presuponía podía ser recuperada en el último cuarto del siglo XIX como un estilo artístico puramente nacional y español (Checa Artazu, 2009).
}

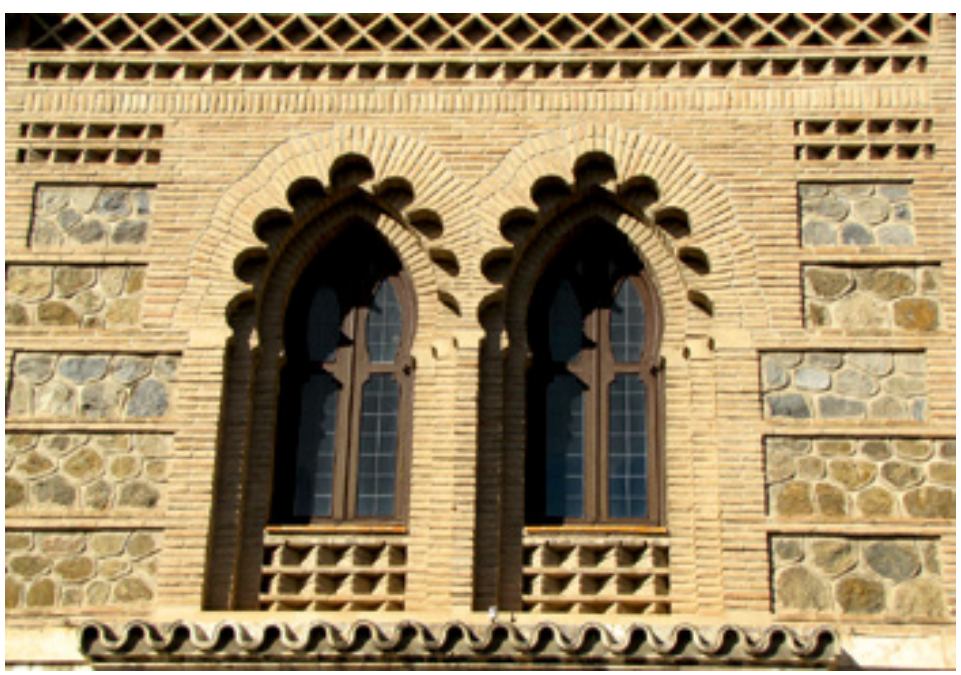

Figura 2. Detalle de fachada de la Estación del Ferrocarril de Toledo. Fuente: Liliana Ruiz Gutiérrez.

En este periodo se erigen varios edificios de estilo mudéjar, tales como la Estación del Ferrocarril de Toledo (1917), construida por Narciso Clavería, y numerosas plazas de toros: Las Ventas en Madrid (1922), diseñada por José Espeliu y Manuel Muñoz Monasterios, La Malagueta en Málaga (1874), de Joaquín Rucoba, y la del Biobio en Gijón (1886-1888), cuyo diseñador fue Ignacio de Velasco. 
Las plazas de toros eran complejos edilicios que contenían espacios como chiqueros, corrales, corralizas, pasillos de conexión, espacios de administración, capilla y enfermería, entre otros. Conviene recordar que los cosos taurinos eran espacios arquitectónicos en evolución formal desde como mínimo el siglo XVI y que en la segunda mitad del XIX observan destacadas mutaciones. Además, las plazas de toros con una mística relacionada con el arte del toreo eran espacios repletos de reminiscencias nacionales y por ende hispánicas, centro neurálgico de la denominada "fiesta nacional", fiesta colectiva que une a la sociedad alrededor del toro y el torero y que había llegado a ser elevada a la categoría de caja de resonancia de los supuestos valores idiosincráticos de España (Checa Artazu, 2009).

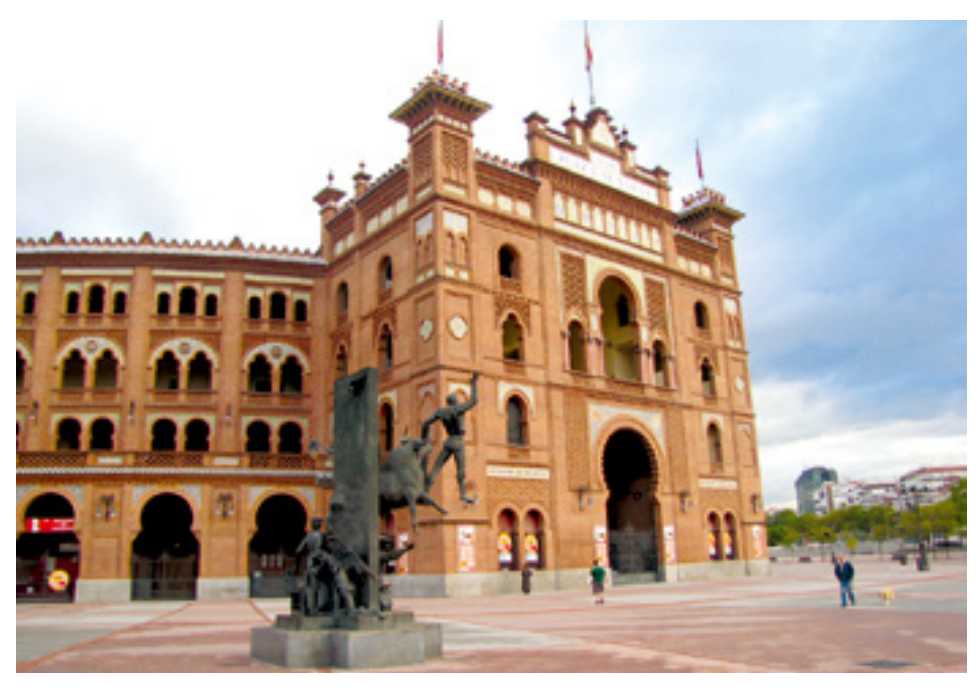

Figura 3. Plaza de Toros de Las Ventas, Madrid.

Fuente: Liliana Ruiz Gutiérrez.

\section{LA ADAPTACIÓN A NUEVAS FUNCIONES}

Con la llegada del siglo XX, el uso de los edificios destinados a los toros experimentó transformaciones. Si bien a lo largo de la historia estos lugares no sólo han albergado el espectáculo taurino sino también otras actividades culturales, deportivas y recreativas, la especificidad de su arquitectura y el cambio en las condiciones culturales de las poblaciones donde se localizan motivaron la aparición de proyectos 
de intervención para recibir nuevas funciones. En algunos casos, tales propuestas han sido centro de polémica por el alto valor patrimonial de las estructuras y el riesgo que implica su paso para fines diametralmente opuestos, pues sus características formales, históricas o simbólicas pueden verse en riesgo.

Sin embargo, tales planteamientos buscan adaptar estos edificios a las condiciones actuales de la sociedad, de modo que su modernización estructural les brinde otras posibilidades y les permita recibir distintos espectáculos y usos articulados a su entorno inmediato, para convertirse así en polos de desarrollo e integración urbana.

Dentro de los proyectos controvertidos desarrollados recientemente en España se describen con brevedad los de las plazas Santa Cruz de Tenerife y Las Arenas.

La plaza de toros Santa Cruz de Tenerife, construida en 1893 por el arquitecto Antonio Pintor, se caracteriza por el uso de arcos en herradura y almenas. Además de su función principal de albergar corridas, allí se realizaron actividades de boxeo, circo, musicales, carnavales, veladas de lucha y proyecciones cinematográficas, entre otros espectáculos. Hasta 1983 recibió actividades taurinas, año en que estas desaparecieron por completo en las Islas Canarias debido a las dificultades económicas de los organizadores y la poca afición local. Después de su abandono a mediados de los años ochenta surgió la iniciativa de modificar su volumetría y dotarla de una cubierta para darle nuevos usos y desarrollar otras prácticas. 
Por su parte, la Plaza de Toros Las Arenas en Barcelona, erigida en los límites del ensanche barcelonés, fue diseñada y construida por Augusto Font y Carreras (18451924) gracias a los aportes privados del banquero Josep Masans y Rof. Inaugurada en 1900, se considera un híbrido entre el modernismo catalán y el neomudejar, con ladrillo a la vista y cerámica como elementos constitutivos de los arcos de herradura y de la fachada en general. Su gran tamaño y características formales le permitieron realizar otras actividades festivas, culturales, políticas y sociales, en las que se buscaba el mayor aprovechamiento económico. En 1977 cesaron las corridas allí, debido al decaimiento de éstas en Cataluña, con lo cual también disminuyeron otros eventos masivos. Como consecuencia, cayó en un alto estado de deterioro.

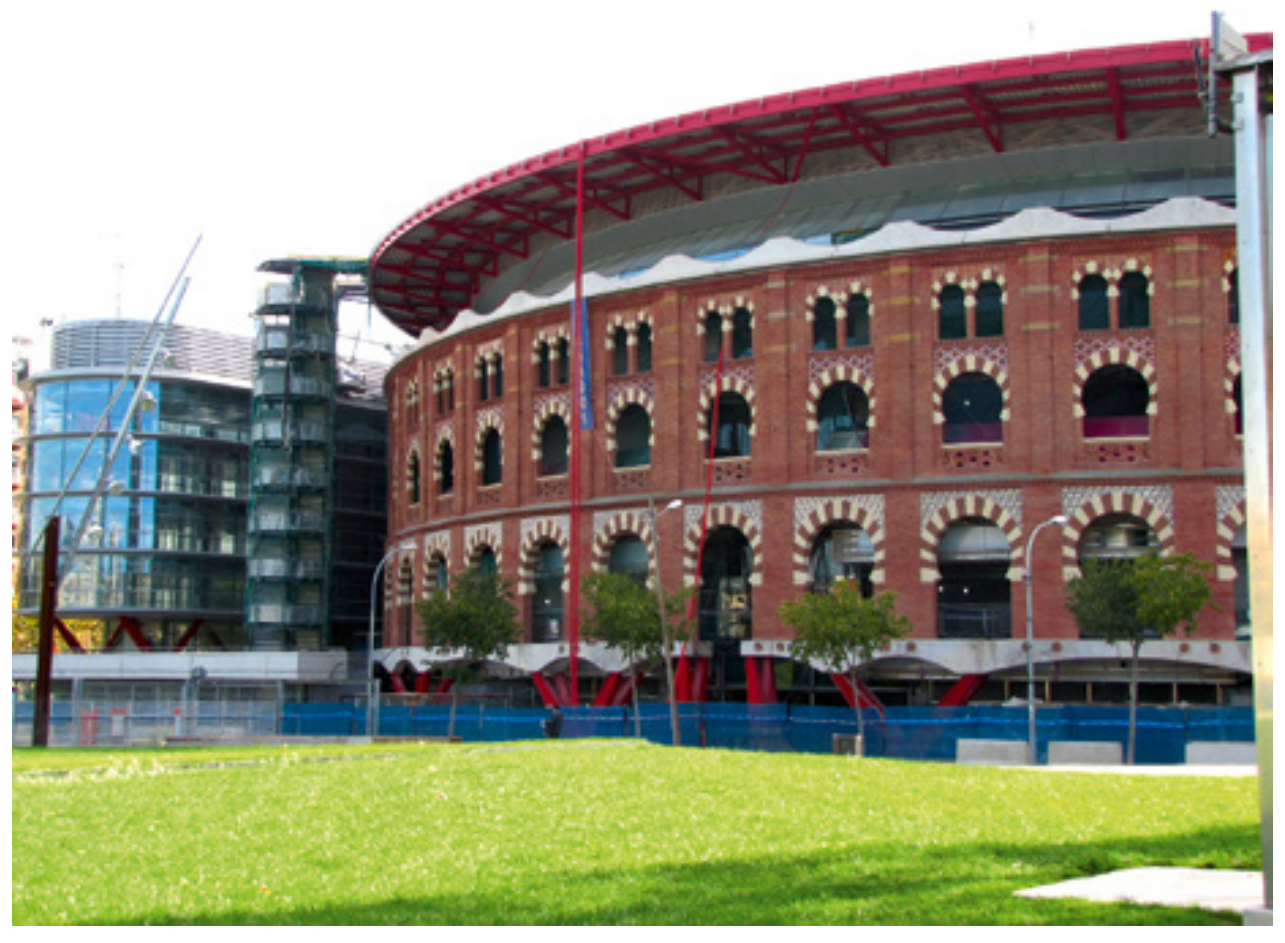


En 1999 una organización privada encargada de la gestión de la plaza propuso una remodelación, idea materializada en 2002 con el proyecto de Richard Rogers y la oficina de arquitectos barceloneses Alonso y Balaguer Asociados. Se buscó aprovechar económicamente el otrora escenario de la tauromaquia y conciliarlo con actividades lúdicas y cívicas. Para esto, se planteó preservar la fachada neomudéjar y el vaciado interior, reforzar la estructura portante e instalar una cubierta. Así se logró el espacio lúdico-comercial hoy en día integrado a las dinámicas urbanas.

\begin{abstract}
Desde el momento que Richard Rogers inició el proyecto de este centro lúdico-comercial presupuso la preservación de la fachada neomudéjar de la plaza. Una presunción que coincidía con la obligación legal de salvaguarda de la fachada como marcaba su nivel de protección patrimonial desde 1993 pero que para el arquitecto británico sirvió de excusa para lanzarse un reto técnico, imposible unos años antes. ¿Era posible conservar la fachada y mantenerla levitando durante gran parte de la ejecución de la obra? Rogers, asociado con el estudio Alonso y Balaguer, asumió ese reto que en parte permitía una cierta lectura amable de la arquitectura a transformar y que sobretodo la incardinaba en la evolución urbana que estaba sufriendo el entorno. Uno de los arquitectos participantes, Luis Alonso, en unas declaraciones mencionaba los motivos: "Desde un primer momento nos fijamos una referencia importantísima: para nosotros Barcelona es un ejemplo en el mundo de respeto hacia el pasado combinado con una apuesta de vanguardia. En Las Arenas creímos muy oportuno desde un principio intentar mantener una fachada que, aun no teniendo un gran valor arquitectónico, llevaba anclada en la retina de los barceloneses toda la vida" (Checa Artazu, 2009).
\end{abstract}

\title{
LA TAUROMAQUIA EN COLOMBIA
}

La fiesta brava llegó a América con la Conquista española. En el actual territorio colombiano se tiene referencia de una primera corrida en 1532, en el Darién, como parte de los festejos para recibir al gobernador Julián Gutiérrez.

Durante la Colonia, las actividades taurinas se consideraban parte fundamental de las fiestas civiles y religiosas. Eran organizadas por los cabildos, los cuales solicitaban toros a los hacendados de cada región. En la plaza mayor de los municipios se levantaban estructuras temporales en madera y en puntos específicos se instalaban palcos para las familias más prestantes de la sociedad de la época. 
La vida durante la Conquista discurrió en fiestas a manera de capeas y más tarde, durante la Colonia, igualmente en cosos de guadua o de talanquera y carros de labor, como acostumbraban y acostumbran los españoles en la península en las fiestas de sus patronos. Durante la Colonia las fiestas de toros en Colombia estaban relegadas a lo de toros y cucañas con un sentido incipiente. No fue la capital el lugar propicio para las corridas, no sabemos por qué, en cambio, en otras ciudades se contaba con "circos" o plazas de toros como Cartagena de Indias, Cali, Pereira y Medellín (Alcázar, 2006, p. 17-18).

Con la creación del Virreinato de la Nueva Granada y la definición de Bogotá como su capital, las corridas aumentaron y se integraron a distintas celebraciones de la corona española, entre ellas el cumpleaños del Rey y la llegada de un nuevo Virrey desde la península. Pedro María Ibáñez, en sus Crónicas de Santafé de Bogotá, relata una impresión de José Celestino Mutis sobre estos espectáculos en la ciudad:

Asistí a la fiesta de toros con cuerda, que fue de lo mejor que llevo vistas en este país. Don Jaime Navarro tendrá ocasión de acordarse en adelante de este día. Fue el caso que habiendo querido seguir la costumbre del país, imitando a los orejones (llaman así a los criollos de los pueblos vecinos y tierra adentro), y a los majitos de este pueblo, cuya gala es salir en tales días montados en sus caballos y rodear y seguir al toro; queriendo pues nuestro don Jaime imitar estos usos, se preparó en su caballo, excediendo tanto su valentía, que se proporcionaba muchas ocasiones de éstas, y en ocasión de estar en la plaza un torito guapo, tuvo la mala suerte de hallarse acometido tan da repente, que no tuvo ocasión de escapar. Pasó toda la desgracia en su caballo, que de la herida murió al siguiente día. Nos ha dado a Su Excelencia y a todos los que conocemos su buen humor, abundante materia para divertirnos con el lance por muchos días (Ibáñez, 1891, p. 165).

La llegada de Carlos III al trono español cambió radicalmente la percepción de esta práctica pues fue condenada al considerarse bárbara y peligrosa, al punto de ser prohibida en las colonias. En la Península Ibérica la nobleza dejó de asistir a las corridas, que se convirtieron en una fiesta para las clases bajas. Se dio entonces una fuerte transformación de esta actividad al reemplazarse su ejecución a caballo por el toreo a pie. Se incorporaron los banderilleros y la muleta como parte integral de la faena. Con la muerte de Carlos III en 1788 se abrió la posibilidad de volver a la participación de todos los sectores sociales en España y de retomar los festejos en América. 
En Bogotá, los escenarios temporales para festejos taurinos solían situarse en la plaza mayor (hoy de Bolívar), el principal sitio para este fin. Posteriormente, también se adecuaron en barrios como San Victorino, Santa Bárbara y Las Nieves.

\section{La diversión de los toros, al igual que la de los gallos, ofrecía varias moda- lidades, quizá la más conocida y frecuente de estas consistió en "correr" toros por las calles y plazas de los barrios. Se realizaba en las noches y a los toros les ataban trapos empapados con materiales inflamables a los cuales se les prendía fuego y se les soltaba, en un "divertimento" llamado la "vaca loca". (Escovar, Mariño \& Peña, 2004, p. 210).}

La primera plaza de toros en la capital, construida por Pietro Cantini, se ubicó en la Huerta de Jaime, hoy Plaza de Los Mártires, con una estructura en madera y se conoció como Plaza de La Bomba. Luego se levantaron otras tantas, entre ellas la instalada en Paiba, la Plaza España y el Circo de San Diego en las inmediaciones del Parque Centenario, que fue reemplazado en varias ocasiones. Tras la construcción de la Santamaría, éste se convirtió en el espacio taurino definitivo.

\section{LA SANTAMARÍA Y SU PRESENCIA EN LA CIUDAD}

El promotor de esta plaza fue el ganadero Ignacio Sanz de Santamaría, quien en 1927 aportó los terrenos donde se erigiría, situados en las inmediaciones del Parque de la Independencia, una zona de piedemonte que para ese momento presentaba un incipiente desarrollo y se encontraba en el límite norte de la ciudad histórica.

La construcción, a cargo de Adonai Martínez y Eduardo Lazcano, se inició en 1928 y fue concluida en 1931. Una parte del área total se asentó sobre roca firme (al oriente), en tanto otra fue elevada y apoyada sobre roca y rellenos (al occidente) para adecuarse a las características topográficas del sector escogido. Originalmente fue una edificación simple, de portada art decó y concreto a la vista, material que empezó a implementarse en el país a principios del siglo XX. 


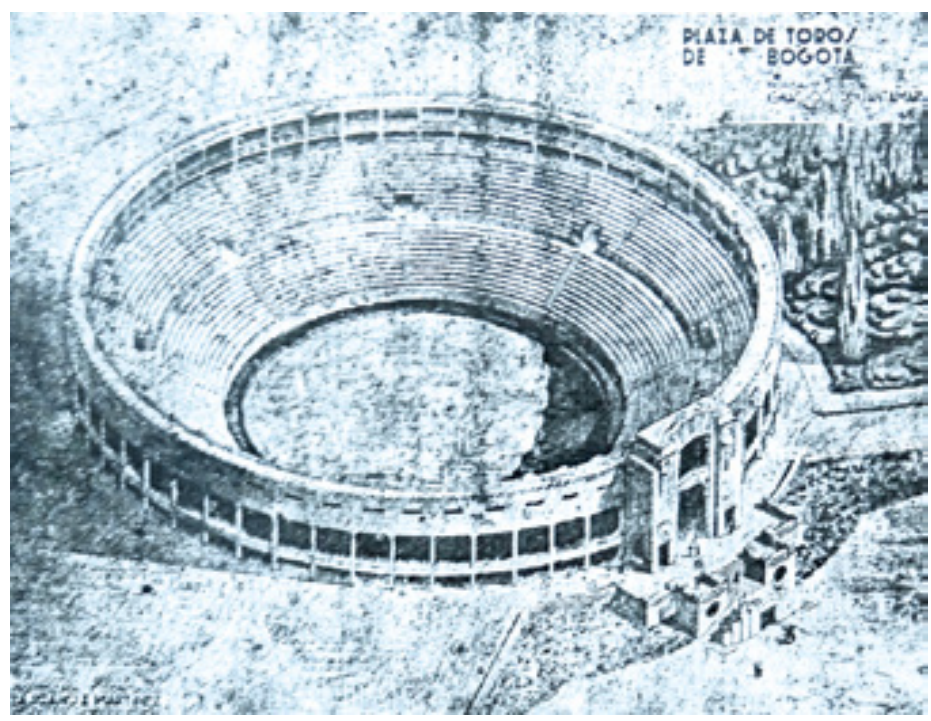

Figura 5. Plaza de Toros de Santamaría, 1931.

Fuente: Museo Taurino.

En 1930, el propietario Santamaría realizó una hipoteca de este predio con el Banco de Bogotá, según lo indicado en la escritura pública 2063 del 25 de septiembre:

Compareció el señor Ignacio Sanz de Santamaría, varón mayor de edad vecino de ésta ciudad a quien conozco personalmente y dijo: Primero: soy deudor del Banco de Bogotá, sociedad anónima domiciliada en esta misma ciudad (...) Constituyo hipoteca a favor del Banco de Bogotá, sobre las siguientes fincas, junto con todas las dependencias, anexidades, edificaciones, útiles, herramientas y demás utensilios a saber: A) Sobre un lote de terreno, ubicado en esta ciudad de Bogotá; que tiene puertas de entrada sobre las calle veintiséis (26) y veintisiete (27) y sobre la carrera quinta ( $\left.5^{a}\right)$, marcadas en sus puertas de entrada con los números treinta y uno (31) y treinta y tres (33) de la calle veintisiete (27), dos E Bis de la calle veintiséis (26), lote en donde actualmente estoy levantando a mis expensas un circo para corridas de toros y espectáculos públicos, edificación que es de cemento armado y la cual construcción queda también incluida en la presente hipoteca (Notaría 4 de Bogotá, 1930, p. 14). 
Ante la imposibilidad de pagar la hipoteca, en 1932 transfirió el predio definitivamente, como se precisa en la escritura pública 2552 del 10 de diciembre:

\begin{abstract}
para pagar todas las deudas determinadas en la cláusula anterior, enajena a título de dación en pago a favor de la Corporación Colombiana de Crédito, los siguientes bienes raíces y muebles: Primero: El lote de terreno ubicado en ésta ciudad de Bogotá que tiene puertas de entrada sobre las calle veintiséis (26) y veintisiete (27) y sobre la carrera quinta (5aㅡ) (...) junto con la edificación levantada en dicho lote que forma el Circo o Plaza de Toros de Bogotá, construcción hecha a expensas del exponente Sanz de Santamaría (...) De ésta enajenación el exponente se reserva según se dirá más adelante el derecho de usufructo durante toda su vida de su palco en el Circo o Plaza de Toros, único palco bajo que existe (Notaría 2 de Bogotá, 1932, p. 6).
\end{abstract}

En 1938 la ciudad compra la plaza y contrata a Santiago de la Mora para terminarla. Este arquitecto diseña una fachada de gran factura en estilo neomudéjar, con ladrillo, arcos de herradura y detalles tipo lacería que cubrieron la estructura en concreto y dieron a la edificación la imagen que hoy en día la identifica.

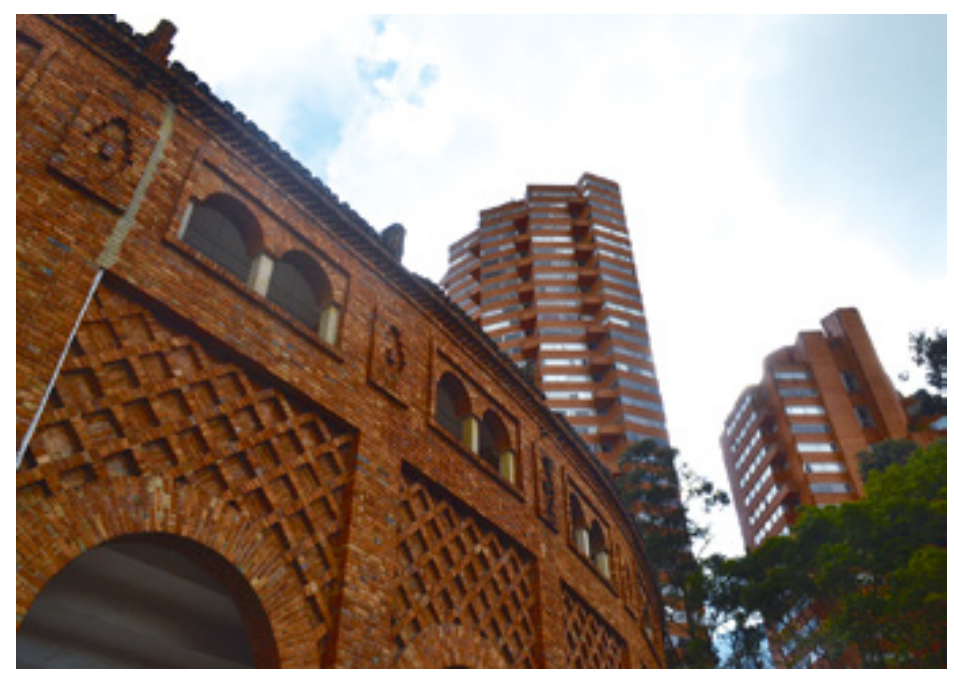

Figura 6. Plaza de Toros de Santamaría en 2014. Fuente: Liliana Ruiz Gutiérrez. 


\section{EL PROYECTO DE INTERVENCIÓN}

Además de corridas de toros, la Santamaría recibió actividades deportivas, artísticas y culturales en periodos distintos a la temporada taurina. Así, varias funciones del Festival Iberoamericano de Teatro de Bogotá se realizaron en la plaza, que también recibió personajes como Mohamed Ali, Libertad Lamarque y Cantinflas.

En 2012 se restringió el uso taurino y se fortalecieron las actividades culturales y deportivas, de modo que la ciudadanía siguió disfrutando de este espacio. Sin embargo, su falta de mantenimiento y la complejidad de su construcción hicieron necesaria una evaluación de su condición de estabilidad estructural, así como de las afectaciones producidas por el uso y la permanencia a la intemperie, entre otros problemas. Se elaboró entonces un proyecto de intervención urbana y recuperación arquitectónica que buscó su integración a las dinámicas actuales de la ciudad.

\section{La condición urbana}

La plaza, localizada en el extremo oriental del centro internacional, está rodeada de bienes de interés cultural del orden nacional y distrital, tales como las Torres del Parque, el Planetario Distrital, el Edificio Antares, el Museo de Arte Moderno, el Parque de la Independencia y el Museo Nacional. Por lo tanto, se trata de un sector con una dinámica cultural y urbana muy representativa para este sector de la ciudad.
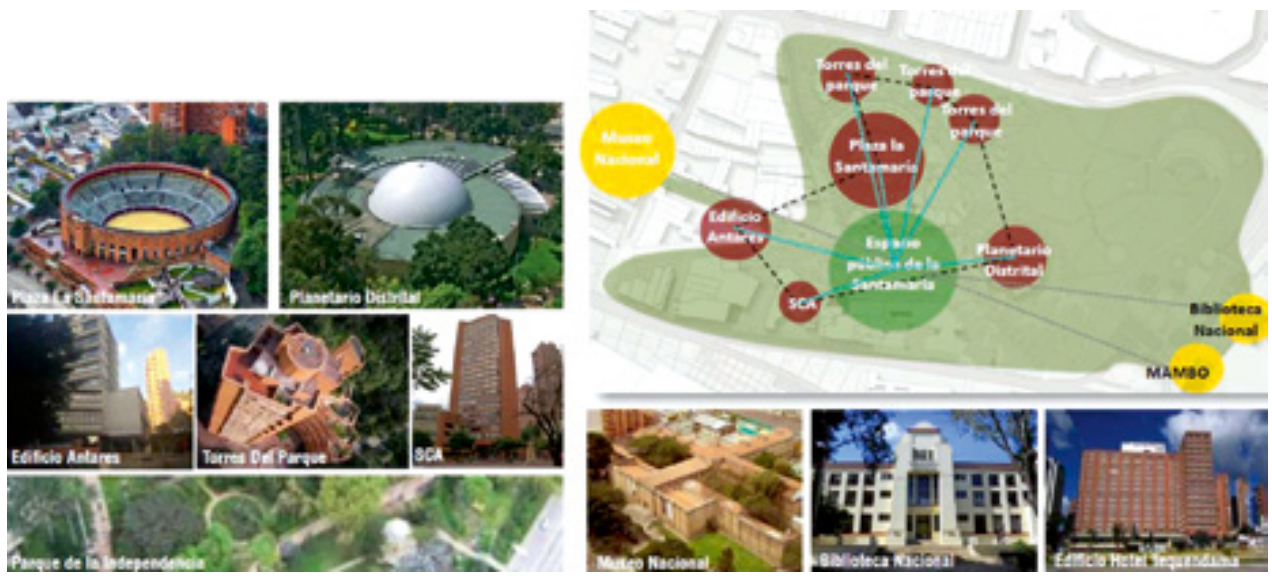

Figura 7. Plaza de Toros y su entorno inmediato con bienes de interés cultural. Fuente: Consorcio V \& P \& Instituto Distrital de Patrimonio Cultural (2013). 
Sin embargo, la falta de iluminación, la deficiencia en el espacio público de calidad y la subutilización del área de parqueadero en el costado occidental, han impedido la vinculación de la plaza al sector. Además, la dificultad de acceso en automóvil y la alta congestión vehicular son limitantes para su desarrollo futuro.

La necesidad de incorporar la edificación a esta zona de alto valor patrimonial determinó el modo de actuación del proyecto urbano. Este planteó la intervención de la zona comprendida entre la Carrera 7, en el acceso al Planetario, la Calle 27 hasta la Carrera 5 y la conexión de la plaza con el Museo Nacional, como también el desarrollo de una plazoleta de acceso en el área del parqueadero.

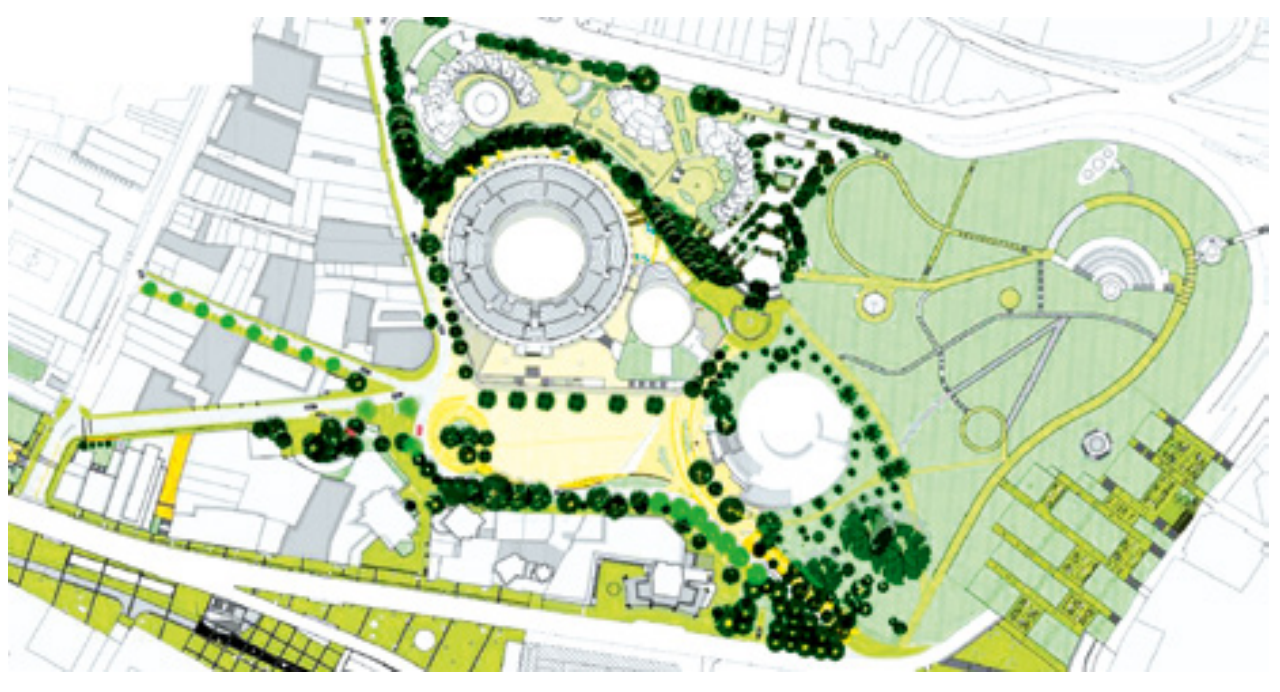

Figura 8. Propuesta de intervención urbana. Fuente: Consorcio V \& P \& Instituto Distrital de Patrimonio Cultural (2013).

Así mismo, define unos lineamientos de intervención que corresponden a:

- Consolidar una unidad morfológica y funcional, la cual incluye el Parque de la Independencia, las Torres del Parque y la plaza de toros como una sola pieza urbana.

- Contribuir al reconocimiento de los valores patrimoniales de los bienes de interés cultural presentes en el sector y generar espacios complementarios de articulación urbana. 
- Consolidar el carácter cívico y cultural de la Santamaría mediante la generación de un espacio polivalente que se articule con los usos y actividades del contexto.

- Consolidar espacios seguros, confortables y funcionales, acordes a la dinámica dotacional y del conjunto, que articulen distintos flujos y privilegien el peatonal.

\section{La recuperación de la Santamaría}

Con el proyecto de intervención se buscó la restauración integral de la edificación, declarada bien de interés cultural de la nación según el Decreto 2390 de 1984. Para tal fin, se pretendió dotarla de los elementos tecnológicos necesarios para albergar actividades culturales y deportivas. Se planteó su reforzamiento estructural, dada su alta vulnerabilidad sísmica a causa de la afectación en algunos aceros de la estructura portante y la disminución en la resistencia del concreto.

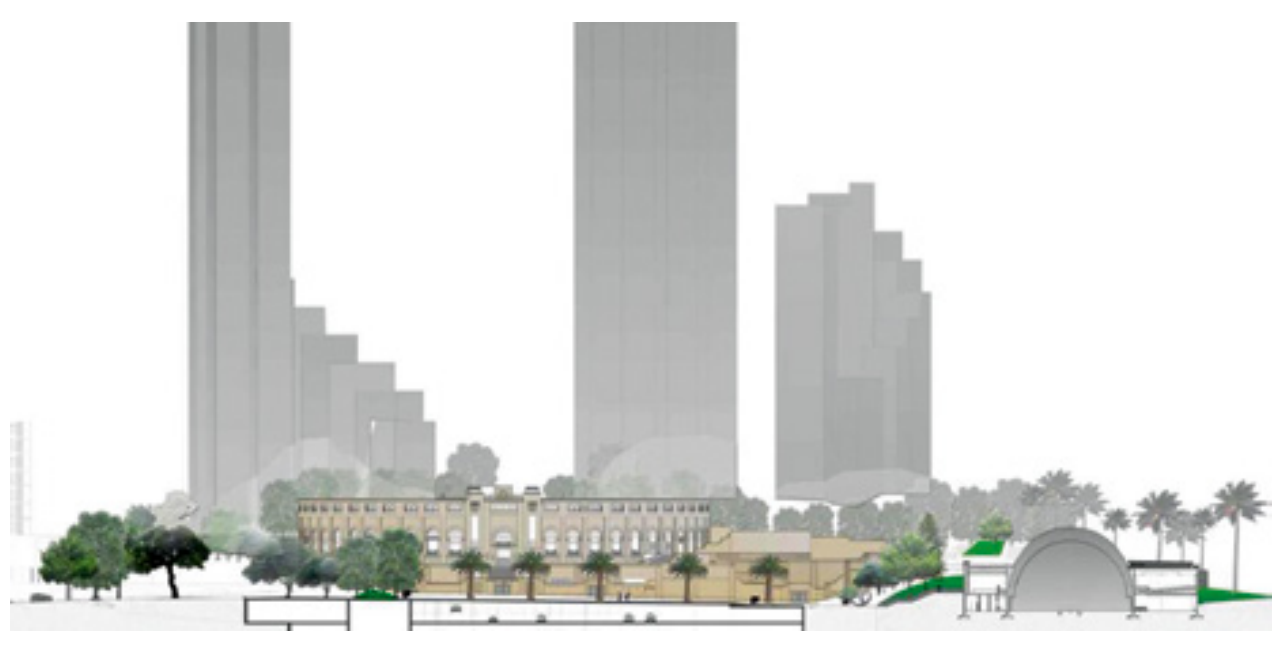

Figura 9. Perfil general del proyecto de intervención. Fuente: Consorcio V \& P \& Instituto Distrital de Patrimonio Cultural (2013).

El Contrato de Consultoría No. 119 de 2013 (Consorcio V \& P \& Instituto Distrital de Patrimonio Cultural) definió estos lineamientos de intervención:

- Reconocimiento y fortalecimiento de la edificación como nodo cultural de la ciudad. 
- Generación de espacios de continuidad urbana entre el Parque de la Independencia y la Santamaría, de manera que ésta pueda recorrerse a diario sin importar si se efectúa o no alguna actividad en su interior.

- Multiplicidad de usos. Al respecto, se propuso potencializar las condiciones técnicas de la arena, con iluminación general y especializada para eventos, sonido y rutas de evacuación. Asimismo, recuperar los antiguos corrales para obtener un escenario alterno destinado a actos culturales de pequeña escala. La temporada de toros, reanudada en 2017, sólo se efectúa en enero y febrero, de modo que es posible adaptar corrales temporales durante estos meses, los cuales podrán desmontarse después de este evento para permitir la realización de otras actividades.

El proyecto se concibió para ser desarrollado en cuatro etapas: 1) reforzamiento estructural; 2) adecuación funcional y restauración integral; 3) intervención de las áreas de ronda y libres al interior del predio; 4) recuperación del espacio público.

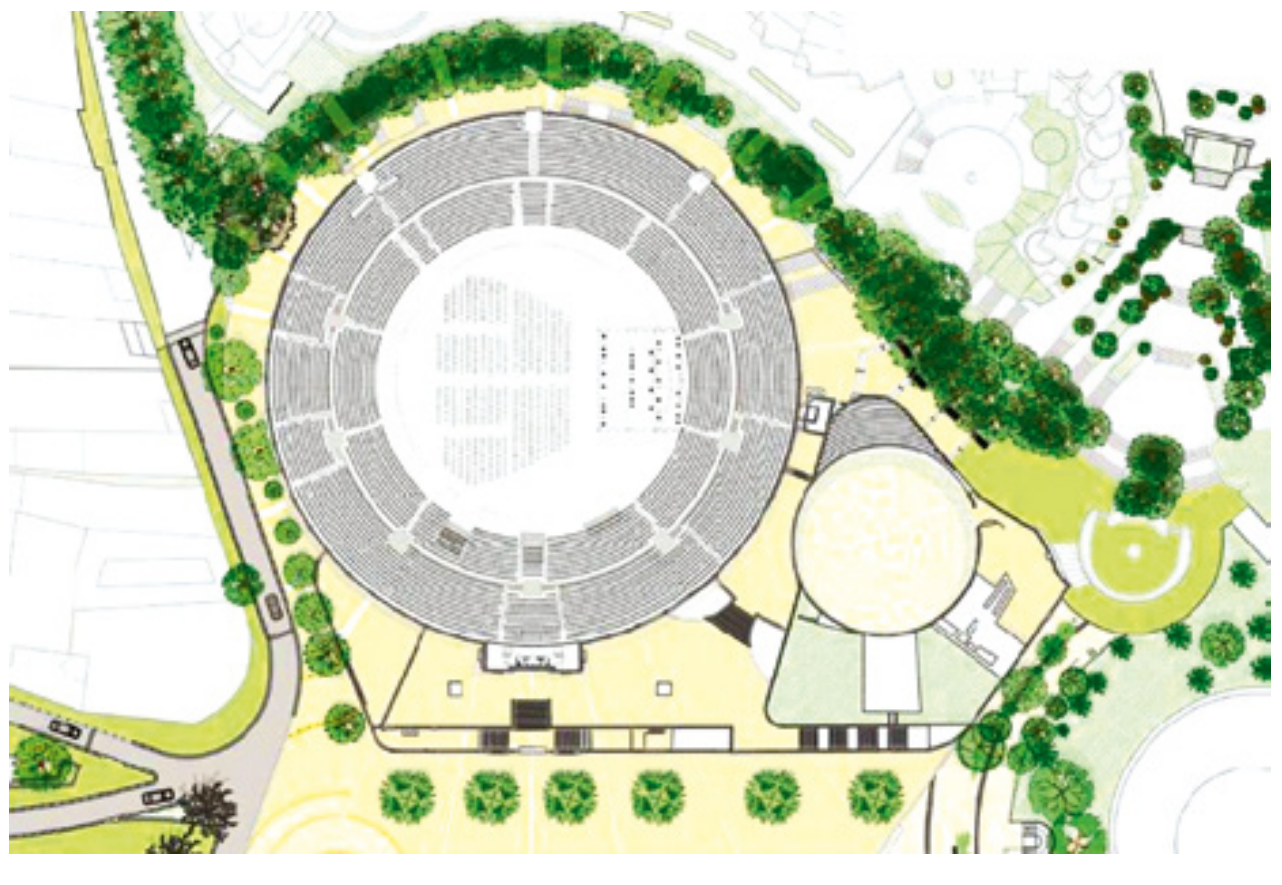

Figura 10. Planta general del proyecto de intervención. Fuente: Consorcio V \& P \& Instituto Distrital de Patrimonio Cultural (2013). 
A partir de esta articulación de un proyecto arquitectónico con una transformación de la imagen de un sector, se apuntó a contribuir al mejoramiento de la ciudad y a poner en valor estructuras y zonas patrimoniales de características singulares. Se dio respuesta así al interrogante sobre cómo un edificio público puede insertarse armónicamente en un contexto y fortalecer la memoria colectiva de la ciudadanía.

\section{REFLEXIONES FINALES}

A lo largo de este documento se ha presentado el abordaje que desde una consultoría pública se hizo en torno a una edificación emblemática de Bogotá. La reflexión surgió de la preocupación por su estado de conservación y frente a la necesidad de adecuarla a las condiciones actuales, en vista de los cambios de uso y ante la pertinencia de buscar su sostenibilidad y apropiación social.

El estudio contempló un análisis detallado (sobre más de $13.000 \mathrm{~m}^{2}$ ) del estado de la plaza. Quizá sea una de las mayores edificaciones patrimoniales evaluadas minuciosamente en Bogotá, no sólo por su área sino también por su relevancia histórica y cultural.

En el desarrollo de la consultoría se agudizó la discusión sobre la continuidad de la tauromaquia en la capital y se definieron dos posiciones diametralmente opuestas. Por una parte, los amantes de esta actividad y la Corporación Taurina la defendieron como un elemento de la cultura bogotana. Por otro lado, los ambientalistas y la administración distrital del momento la consideraron una tortura cruel y abogaron por la protección de los derechos de los animales. Si bien el debate se mantiene, lo cierto es que en Bogotá y otras ciudades ha disminuido el interés por la práctica. Al respecto, en una columna del periódico El Tiempo se hizo un paralelo con lo que ocurre en Medellín: 
La controversia llegó hasta la Corte Constitucional. En 2013, bajo el argumento del libre derecho a la expresión artística de la Corporación Taurina de Bogotá, la Sentencia T-296/13 obligó a la ciudad a reabrir la plaza para el uso taurino:

\section{Ni el marco legal para la realización de la tauromaquia, el Reglamento Nacional Taurino (L.916/04), ni los condicionamientos introducidos por la Corte Constitucional (Sentencia C-666/10), facultan a la administración distrital para imponer la alteración de la estructura del espectáculo taurino para eliminar la muerte del toro, como tampoco para impedir la realización de espectáculos taurinos que cumplieran los requisitos constitucionales y legales (p. 7).}

Es importante señalar que los cambios de uso, como aquí se ha expuesto, han sido un común denominador en estas edificaciones, pues aunque en su gran mayoría se erigieron para ser destinadas principalmente a la tauromaquia, durante su historia también han albergado eventos variados, masivos, artísticos y culturales.

La polémica debe centrarse en la importancia de los edificios patrimoniales como generadores de identidad e integrantes de la historia de la ciudad, con potencial para el desarrollo urbano y social. Con los años se harán análisis sobre las sociedades actuales y emergerán otras reflexiones en torno a la tauromaquia. Esas visiones, distintas a las del momento de aparición de la práctica y a las observadas a lo largo de su existencia, pueden llevar a su transformación o incluso desaparición.

Ejemplo de estos cambios son las luchas de gladiadores durante el Imperio Romano o la protección fortificada de ciudades. Con el tiempo, estas prácticas y funciones, al igual que muchas otras, han desaparecido o se han modificado, pero dejaron en los territorios marcas físicas de singular valor (el Coliseo Romano, numerosas murallas, etc), las cuales se aprecian hoy desde su valor histórico, artístico o técnico, cualidades que se espera conservar y transmitir a las generaciones futuras.

En el caso de la Santamaría, la ejecución del reforzamiento estructural ha dejado en evidencia la manera como fue construida, a partir de un sistema aporticado en concreto, que apenas empezaba a implementarse en el país. A pesar de no contar en su factura con los mejores desarrollos técnicos que este material permite, ha sido un referente para la ciudad y se busca adecuar a los requerimientos de sismorresistencia para esta clase de edificios, además de potencializar sus valores arquitectónicos, simbólicos e históricos, consolidándose como un referente en la memoria de propios y extraños en Bogotá. 


\section{REFERENCIAS BIBLIOGRÁFICAS}

Alcázar, P. (2006). Plazas de Toros de Colombia. Ciudad: Madrid-España. Agualarga Editores S.L.

Checa Artazu, M. (2009). Refuncionalizaciones polémicas, plazas de toros y arquitectura neomudéjar: algunos ejemplos en España. Revista Bibliográfica de Geografía y Ciencias Sociales, (Vol XIV, No. 839). Recuperado de http://www.ub.edu/geocrit/b3w-839.htm

Consorcio V \& P \& Instituto Distrital de Patrimonio Cultural. Contrato de Consultoría 119 de 2013.

Corte Constitucional. (2013). Sentencia T-296/13.

Escovar, A., Mariño, M. \& Peña, C. (2004). Atlas histórico de Bogotá, 1538-1910. Bogotá: Corporación La Candelaria.

Gómez, S. (2015). Tauromaquia: muerte lenta pero segura. El Tiempo. Recuperado de http://www.eltiempo. com/opinion/columnistas/tauromaquia-muerte-lenta-pero-segura-sonia-gomez-gomez-columnistaopinion/15233516

Ibáñez, P. M. (1891). Crónicas de Santafé de Bogotá. Recuperado de http://www.banrepcultural.org/blaavirtual/historia/cronidos/capxxiii.htm

Notaría 2 de Bogotá. (1932). Escritura pública 2552 del 10 de diciembre.

Notaría 4 de Bogotá. (1930). Escritura pública 2063 del 25 de septiembre.

Pérez de Lama Halcón, J. (1994). Plazas de toros. Revista de Estudios Taurinos, 1, 181-218.

Schwartz, C. (1986). Cubierta de la Plaza de Toros de Santa Cruz de Tenerife, España. Informe de construcción nro. 381. Recuperado de http://informesdelaconstruccion.revistas.csic.es/index.php/informesdelaconstruccion/ article/viewFile/1758/1945 